\title{
Spontaneous growth of gauge fields in gravity through the Higgs mechanism
}

\author{
Fethi M. Ramazanoğlu \\ Department of Physics, Koç University, Rumelifeneri Yolu, 34450 Sariyer, Istanbul, Turkey
}

(Received 10 April 2018; published 6 August 2018)

\begin{abstract}
We introduce gravity theories featuring spontaneously growing gauge fields where the growth is due to the Higgs mechanism. The underlying physics is inspired by the spontaneous scalarization phenomena in scalar-tensor theories. The tachyonic instability that causes the growth in spontaneous scalarization and its analog for vector fields is introduced not as an explicit potential term, but through a scalar coupling using a Higgs field. The resulting theories are distinct from previous examples of spontaneous tensorization in that they respect the gauge symmetry at the level of the action. Our results are valid for both Abelian and nonAbelian gauge theories, and this is the first study of the spontaneous growth of the latter. We discuss astrophysical implications of these theories and argue their relevance, especially in the strong gravity regime.
\end{abstract}

DOI: 10.1103/PhysRevD.98.044013

\section{INTRODUCTION}

Scalar-tensor theories are alternative theories to general relativity (GR) where gravitation is governed by additional scalar fields together with the metric [1]. When certain coupling functions of these theories are appropriate, the solution with vanishing scalar fields that corresponds to GR becomes unstable inside a neutron star (NS) [2]. Arbitrarily small scalar-field perturbations exponentially grow due to a a tachyonic instability, but the growth eventually stops to form a scalar cloud around the star. The final stable field configuration can routinely attain large amplitudes; hence, deviations from GR are prominent near the NS. At the same time, the scalar dies off with distance from the star. This presents an ideal scenario from an observational point of view: weak-field tests of gravity are satisfied in the far field, and large deviations in strong gravity provide a relatively easy target for gravitational wave detection, which is precision-limited $[3,4]$.

The central idea of spontaneous scalarization, a tachyonic instability of the zero-field solution, has been recently generalized to other fields such as vectors [5]. NSs with vector clouds around them have similar properties to scalarized stars; hence, they have the same appeal in terms of observations. However, the gauge symmetry we find in the known vector fields in the universe is explicitly broken in the actions of these new theories. This is not necessarily a problem as long as we remain in the classical realm, but it is an unnatural quality in light of our knowledge about fundamental fields.

In this study we present a new mechanism for spontaneous growth of gauge fields, Abelian or non-Abelian, that respects the gauge symmetry at the level of the action. The essence of our work is a simple idea that features the Higgs mechanism $[6,7]$. The tachyon of spontaneous scalarization arises from a local effective mass term that has the "wrong" sign. This means there are modes with imaginary energy that grow in time instead of oscillate, and form an instability. A Higgs coupling term also generates mass for gauge fields, so it can also incite tachyons and lead to spontaneous growth with a similar "wrong" sign choice. We name the resulting phenomena "Higgs-based spontaneous growth." The required nonzero expectation value of the Higgs field can be obtained through a Mexican hat potential term or the recently studied gravitational Higgs mechanism [8]. We will present spontaneous growth theories for each case.

We will discuss the astrophysical relevance of Higgsbased spontaneous growth in comparison to what we already know about spontaneous scalarization and its generalizations. An important point is that the equation of motion (EOM) of the Higgs field itself is also modified in these new theories in a similar way to ghost-based spontaneous scalarization theories [9]. This has important implications for NS structure.

Section II is a summary of spontaneous scalarization and its various generalizations. Section III is the heart of this study where we present Higgs-based spontaneous growth of Abelian and non-Abelian field theories. Section IV contains our comments on the results, comparison to the existing literature, and observational prospects of Higgsbased spontaneous growth. We use gravitational units $G=c=1$.

\section{SPONTANEOUS SCALARIZATION AND ITS GENERALIZATIONS}

The simplest example of spontaneous growth in gravity and the inspiration for all other spontaneously growing fields is "spontaneous scalarization" [2] described by the action 


$$
\begin{aligned}
& \frac{1}{16 \pi} \int d V R-\frac{1}{4 \pi} \int d V[\overbrace{\frac{1}{2} g^{\mu \nu} \partial_{\mu} \phi \partial_{\nu} \phi}^{T_{\phi}} \overbrace{\frac{1}{2} m_{\phi}^{2} \phi^{2}}^{V_{\phi}}] \\
& +S_{m}\left[f_{m}, A^{2}(\phi) g_{\mu \nu}\right]
\end{aligned}
$$

where $g_{\mu \nu}$ is the metric, $\phi$ is a real scalar field, $m_{\phi}$ is the parameter of the scalar potential which can be interpreted as mass in the frame of $g_{\mu \nu}$, and $d V=d^{4} x \sqrt{-g} . S_{m}$ is the matter action, $f_{m}$ representing all matter degrees of freedom. The alternative nature of the theory to GR arises from the nonminimal coupling in $S_{m}$ where matter directly interacts with the conformally scaled metric $\tilde{g}_{\mu \nu}=A^{2}(\phi) g_{\mu \nu}$. The reference frame defined by this metric is traditionally called the Jordan frame, while that of $g_{\mu \nu}$ is the Einstein frame. We will quickly summarize the interesting properties of this action, but a more detailed account with our particular emphases can be found in [10].

If the conformal scaling has the specific form $A(\phi)=$ $e^{\beta \phi^{2} / 2}$ or more generally has the Taylor expansion $A(\phi)=$ $1+\beta \phi^{2}+\cdots$, it turns out the $\phi=0$ solution is unstable in the presence of matter for low Fourier modes, i.e., any small perturbation initially grows exponentially [2]. The reason for this can be quickly identified in the equation of motion (EOM)

$$
\begin{aligned}
\square_{g} \phi & =\left(-8 \pi A^{4} \frac{d(\ln A(\phi))}{d\left(\phi^{2}\right)} \tilde{T}+m_{\phi}^{2}\right) \phi \\
& \approx\left(-4 \pi \beta \tilde{T}+m_{\phi}^{2}\right) \phi
\end{aligned}
$$

where $\tilde{T}$ is the trace of the stress-energy tensor in the Jordan frame, and we linearized the equation around $\phi=0$ in the second line. It is clear that the scalar behaves as if it has an effective mass-square $m_{\text {eff }}^{2}=\left(-4 \pi \beta \tilde{T}+m_{\phi}^{2}\right)$, and $m_{\text {eff }}^{2}<0$ for $\beta<0$ since $\tilde{T}<0$ in most cases. A scalar with imaginary mass is called a "tachyon." The dispersion relation $E^{2}=m^{2}+k^{2}$ implies that the energies of low $k$ modes are also imaginary; hence, these modes grow exponentially in time rather than oscillate.

When $\phi$ grows large enough, $A^{4}$ factor in the EOM kills the negative mass-square term and ensures that the growth eventually stops. For the natural choice where $|\beta| \sim 1$, the relevant length scales mean that NSs possess scalar clouds, but less compact stars do not. The scalar amplitude is generically large inside and near the star; hence, this theory promises relatively easily observable deviations from GR. On the other hand, the scalar dies off with distance from the star in any physical solution, ensuring agreement with weak-field tests of gravity. These aspects of spontaneous scalarization have made it popular for strong gravity physics and gravitational wave science.

Let us examine why we chose $A(\phi)=e^{\beta \phi^{2} / 2}$, which will help us understand other theories of spontaneous growth later. When we vary the action with respect to $\phi$, the scalar potential $\left(V_{\phi}\right)$ contribution to the equation of motion is $m_{\phi}^{2} \phi$, the mass-square term. If $A(\phi)$ has a form similar to $V_{\phi}$, its variation also brings a mass-square term. In short, $A(\phi)$ can be used to generate mass if it has the correct functional form. This mass can be made negative, i.e., tachyonic, by choosing the "wrong" sign for the coefficients in the Taylor expansion of $A$.

Our understanding of the physics of spontaneous scalarization recently gave rise to generalization of this phenomena in two directions. First, note that there is nothing special about the nature of the field itself in our explanation for spontaneous growth. Thus, if we have another field, e.g., a vector, and introduce a nonminimal matter coupling that provides an effective mass term with the appropriate sign, we expect to observe spontaneous vectorization. This idea was realized using the action [9]

$$
\begin{aligned}
& \frac{1}{16 \pi} \int d V R-\frac{1}{16 \pi} \int d V\left[F^{\mu \nu} F_{\mu \nu}+2 m_{X}^{2} X^{\mu} X_{\mu}\right] \\
& \quad+S_{m}\left[f_{m}, A_{X}^{2}(\eta) g_{\mu \nu}\right], \quad \eta=g^{\mu \nu} X_{\mu} X_{\nu}
\end{aligned}
$$

where $X$ is a vector field, $F_{\mu \nu}=\nabla_{\mu} X_{\nu}-\nabla_{\nu} X_{\mu}$, and $A_{X}=$ $e^{\beta_{X} \eta / 2}$. The EOM

$$
\nabla_{\rho} F^{\rho \mu}=\left(-8 \pi A_{X}^{4} \Lambda \tilde{T}+m_{X}^{2}\right) X^{\mu}
$$

easily shows the imaginary effective mass, hence the tachyon and spontaneous growth. Note the choice of $A_{X}$ that is inspired from spontaneous scalarization.

The second path to generalize spontaneous growth takes advantage of the fact that the nature of the instability is not critical either, i.e., as long as the zero-field solution is unstable, the field grows spontaneously. For example, just as the tachyon effectively modifies the potential term $V_{\phi}$ in Eq. (1), one can have the "wrong" sign in the kinetic term $T_{\phi}$ due to derivative coupling terms in the matter action. Such an instability is called a "ghost," and it can be used to construct a "ghost-based spontaneous scalarization" theory. Concretely, the action [5]

$$
\begin{aligned}
& \frac{1}{16 \pi} \int d V R-\frac{1}{16 \pi} \int d V\left[2 g^{\mu \nu} \partial_{\mu} \phi \partial_{\nu} \phi+2 m_{\phi}^{2} \phi^{2}\right] \\
& \quad+S_{m}\left[f_{m}, A_{\partial}^{2}(\kappa) g_{\mu \nu}\right], \quad \kappa \equiv g^{\mu \nu} \partial_{\mu} \phi \partial_{\nu} \phi
\end{aligned}
$$

leads to the EOM

$$
\left(-4 \pi A_{\partial}^{4} \beta_{\partial} \tilde{T}+1\right) \square_{g} \phi=m_{\phi}^{2} \phi
$$

for $A_{\partial}(\kappa)=e^{\beta_{\partial} \kappa / 2}$. Alternatively, we can move the negative sign to the left-hand side of Eq. (6) and obtain a tachyonic EOM

$$
\square_{g} \phi=\left(-4 \pi A_{\partial}^{4} \beta_{\partial} \tilde{T}+1\right)^{-1} m_{\phi}^{2} \phi
$$


Thus, the two spontaneous scalarization theories behave similarly at the level of the EOM. Many of the conclusions for this action are similar to the theory of Eq. (1). When we need to distinguish the two forms of spontaneous scalarization, we will call the theory of Eq. (1) "tachyon-based spontaneous scalarization."

There are also important distinctions between tachyonand ghost-based spontaneous scalarization. The effective mass term $\left(-4 \pi A_{\partial}^{4} \beta_{\partial} \tilde{T}+1\right)^{-1} m_{\phi}^{2}$ can possibly diverge in ghost-based spontaneous scalarization, leading to peculiar changes in the NS structures. We will discuss this issue further in the discussion section. We consider ghost-based spontaneous scalarization as a purely classical modification to general relativity, which itself is a classical field theory. However, if one wishes to quantize this theory, more care is needed compared to tachyon-based spontaneous scalarization due to the particularly dangerous behavior of ghosts. Like the tachyon, it is also possible, but not explicitly demonstrated, that the ghost is also regularized, i.e., the instability shuts off as the ghost field grows [9]. If this is the case, we might also have some improvement of the quantum behavior as in ghost condensation [11]. A thorough study of this question is beyond the scope of this paper.

We can also combine the two ideas to obtain a theory of "ghost-based spontaneous vectorization" which has the action [5]

$$
\begin{gathered}
\frac{1}{16 \pi} \int d V R-\frac{1}{16 \pi} \int d V\left[F^{\mu \nu} F_{\mu \nu}+2 m_{X}^{2} X^{\mu} X_{\mu}\right] \\
+S_{m}\left[\psi_{m}, A_{F}^{2} g_{\mu \nu}\right], \quad A_{F}=e^{\beta_{F} F^{\mu \nu} F_{\mu \nu} / 4},
\end{gathered}
$$

and the EOM

$$
\left(-4 \pi A_{F}^{4} \beta_{F} \tilde{T}+1\right) \nabla_{\rho} F^{\rho \mu}=m_{X}^{2} X^{\mu} .
$$

"Spontaneously growing non-Abelian field theories" have not appeared in the literature to the best of our knowledge. They can be formulated in a similar manner to spontaneous vectorization as

$$
\begin{aligned}
S= & \frac{1}{16 \pi} \int d V R-\frac{1}{16 \pi} \int d V\left(F^{a \mu \nu} F_{\mu \nu}^{a}+2 m_{W} W_{\mu}^{a} W^{a \mu}\right) \\
& +S_{m}\left[\psi_{m}, A_{W}^{2} g_{\mu \nu}\right]
\end{aligned}
$$

where

$$
F_{\mu \nu}^{a}=\nabla_{\mu} W_{\nu}^{a}-\nabla_{\nu} W_{\mu}^{a}+e f^{a b c} W_{\mu}^{b} W_{\nu}^{c},
$$

$a, b, c$ label the vector potential $W_{\mu}^{a}$ and the generators $T^{a}$ of the Lie algebra of the gauge group of the theory, $\left[T^{a}, T^{b}\right]=$ $i f^{a b c} T^{c}$ is the defining equation for the structure constants $f$, and $e$ is a coupling constant [6,7]. For example, if the gauge group is $S U(2)$ (a Yang-Mills theory), $T^{a}$ are the Pauli matrices, $f^{a b c}=\epsilon^{a b c}$ and $a=1,2,3$.

$$
A_{W}=\exp \left(\frac{\beta_{W}}{2} W_{\mu}^{a} W^{a \mu}\right)
$$

in analogy to Eq. (3), or

$$
A_{W}=\exp \left(\frac{\beta_{F, W}}{2} F^{a \mu \nu} F_{\mu \nu}^{a}\right),
$$

in analogy to Eq. (8), provide tachyon- or ghost-based versions of the theory, respectively.

\section{SPONTANEOUS GROWTH THROUGH THE HIGGS MECHANISM}

Spontaneous vectorization in Eq. (3) is very simply analogous to spontaneous scalarization in Eq. (1) in terms of mathematical form, and it is not surprising that it was conceived as the first generalization of spontaneous growth beyond scalars. ${ }^{1}$ However, this action explicitly breaks the gauge symmetry

$$
X_{\mu} \rightarrow X_{\mu}-\partial_{\mu} \lambda
$$

that leaves the minimally coupled vector-field action

$$
\frac{1}{16 \pi} \int d V R-\frac{1}{16 \pi} \int d V F^{\mu \nu} F_{\mu \nu}
$$

invariant, where $\lambda$ is a scalar function. The term directly responsible for spontaneous growth, $A_{X}$, breaks the symmetry; hence, it seems explicit symmetry breaking is unavoidable in this formulation. ${ }^{2} A_{F}$ in ghost-based spontaneous vectorization [Eq. (8)] respects the symmetry in Eq. (14); however, the mass term $2 m_{X}^{2} X^{\mu} X_{\mu}$ is necessary in this theory to have spontaneous growth in the EOM [Eq. (9)]. Hence, the gauge symmetry still has to be explicitly broken.

Why are we concerned with the breaking of this symmetry? First, vector fields we know to exist in nature do have gauge symmetry in some form. Second, gauge symmetry is also important for quantization in the nonAbelian case $^{3}[6,7,13]$. These concerns do not invalidate spontaneous vectorization as formulated in Eq. (3) as long as we view it as a classical theory and do not identify $X^{\mu}$ with a known field in nature, i.e., if it is considered to be a yet undiscovered field of gravitational interactions.

\footnotetext{
[12].

${ }^{1}$ Equation (3) was introduced earlier in a cosmological setting

${ }^{2}$ The mass terms $m_{\phi}$ and $m_{X}$ are not necessary for tachyonbased spontaneous growth - they actually inhibit it. However, $m_{\phi}$ is needed for agreement with observations [10], and we expect a similar case for $m_{X}$ [5].

${ }^{3}$ However, note that an explicit mass term is still admissible as long as we remain below the strong coupling scale.
} 
However, lack of symmetry makes this theory less "natural" and weakens the motivation to study it.

Before we conceive a theory of spontaneous growth that respects the gauge symmetry, let us see how we can make a minimally coupled vector field under GR, Eq. (15), massive using the famed Higgs mechanism [6,7]. For the simplest case, let us consider the action of a massless vector together with a massless complex scalar $\Phi$

$$
\begin{aligned}
& \frac{1}{16 \pi} \int d V R-\frac{1}{16 \pi} \int d V F^{\mu \nu} F_{\mu \nu} \\
& +\frac{1}{16 \pi} \int d V\left(2 \overline{D^{\mu} \Phi} D_{\mu} \Phi+2 V(\bar{\Phi} \Phi)\right)
\end{aligned}
$$

Here

$$
D_{\mu} \Phi=\left(\nabla_{\mu}-i e X_{\mu}\right) \Phi
$$

is the gauge-invariant derivative, $e$ is a real coupling constant, and an overbar denotes complex conjugation. The action respects the gauge symmetry through the transformation

$$
X_{\mu} \rightarrow X_{\mu}-\nabla_{\mu} \lambda, \quad \Phi \rightarrow e^{i e \lambda} \Phi .
$$

Where is the mass term? At the most basic level, expanding $D_{\mu}$ brings a Lagrangian density

$$
\mathcal{L}_{X}=-\frac{1}{4} F^{\mu \nu} F_{\mu \nu}-\frac{1}{2}\left(e^{2} \bar{\Phi} \Phi\right) X^{\mu} X_{\mu}+\cdots
$$

Thus, $e^{2} \bar{\Phi} \Phi$ behaves as an effective mass-square term. This does not mean much by itself since linear perturbations of $X_{\mu}$ are still massless around the solution with $X_{\mu}=0=\Phi$. However, the mechanism is completed by the potential

$$
V(\bar{\Phi} \Phi)=\frac{1}{2} \frac{m_{0}^{2}}{u^{2}}\left(u^{2}-\bar{\Phi} \Phi\right)^{2}
$$

for real constants $u$ and $m_{0}$. The scalar behaves as a tachyon around $\Phi=0$

$$
\square \Phi=m_{0}^{2}\left(\bar{\Phi} \Phi / u^{2}-1\right) \Phi+\cdots
$$

with linearized mass $i m_{0}$, but it has a stable equilibrium for $|\Phi|=u$. The stable configuration, whatever $\Phi$ we choose, provides a nonzero value of $\bar{\Phi} \Phi=u^{2}$. Even though the Lagrangian is symmetric, the ground state of $\Phi$ is not, and this phenomenon is called "spontaneous symmetry breaking." Hence, for linear perturbations around the equilibrium values of the fields, $X^{\mu}$ behaves as if it has mass $e u$.

Since spontaneous scalarization in Eq. (1) and vectorization in Eq. (3) are based on mass generation in their essence, the Higgs mechanism immediately suggests a new type of spontaneous growth. Remember that the conformal coupling had a mathematical form similar to the mass potential term of the vector Lagrangian in Eq. (3). Thus, we consider an action where the conformal scaling function has the form that generates the $e^{2} u^{2}$ term in Eq. (16) as

$$
\begin{aligned}
& \frac{1}{16 \pi} \int d V R-\frac{1}{16 \pi} \int d V F^{\mu \nu} F_{\mu \nu} \\
& \quad-\frac{1}{16 \pi} \int d V\left(2 \overline{D_{\mu} \Phi} D^{\mu} \Phi+2 V(\bar{\Phi} \Phi)\right) \\
& \quad+S_{m}\left[\psi_{m}, A_{H}^{2} g_{\mu \nu}\right],
\end{aligned}
$$

where

$$
A_{H}=\exp \left(\frac{\beta_{H}}{2} \overline{D_{\mu} \Phi} D^{\mu} \Phi\right)
$$

Variation of the action leads to

$$
\begin{aligned}
G_{\mu \nu}= & 8 \pi T_{\mu \nu}+T_{\mu \nu}^{\Phi, X}-g_{\mu \nu} V(\bar{\Phi} \Phi) \\
\nabla^{\nu} F_{\nu \mu}= & \left(-4 \pi \beta_{H} \tilde{T} A_{H}^{4}+1\right)\left(e^{2} \bar{\Phi} \Phi X_{\mu}+J_{\mu}^{\Phi}\right) \\
\square \Phi= & {\left[m_{0}^{2}\left(\bar{\Phi} \Phi / u^{2}-1\right)\left(-4 \pi \beta_{H} \tilde{T} A_{H}^{4}+1\right)^{-1}\right.} \\
& \left.+e^{2} X_{\mu} X^{\mu}+2 i e X^{\mu} \nabla_{\mu}+i e \nabla_{\mu} X^{\mu}\right] \Phi
\end{aligned}
$$

where

$$
\begin{aligned}
T_{\mu \nu}= & A_{H}^{2} \tilde{T}_{\mu \nu}-\beta_{H} \tilde{T} A_{H}^{4} \overline{D_{(\mu} \Phi} D_{\nu)} \Phi \\
T_{\mu \nu}^{\Phi, X}= & 2 \nabla_{(\mu} \bar{\Phi} \nabla_{\nu)} \Phi-g_{\mu \nu} g^{\rho \sigma} \nabla_{\rho} \bar{\Phi} \nabla_{\sigma} \Phi \\
& +2 F_{\mu \rho} F_{\nu}{ }^{\rho}-\frac{1}{2} g_{\mu \nu} F_{\rho \sigma} F^{\rho \sigma} \\
& +2 e^{2} \bar{\Phi} \Phi\left(X_{\mu} X_{\nu}-\frac{1}{2} g_{\mu \nu} g^{\rho \sigma} X_{\rho} X_{\sigma}\right) \\
& +2 J_{\mu}^{\Phi} X_{\nu}+2 J_{\nu}^{\Phi} X_{\mu}-2 g_{\mu \nu} g^{\rho \sigma} J_{\rho}^{\Phi} X_{\sigma} \\
J_{\mu}^{\Phi}= & \frac{i e}{2}\left(\bar{\Phi} \nabla_{\mu} \Phi-\Phi \nabla_{\mu} \bar{\Phi}\right) .
\end{aligned}
$$

If matter is not very relativistic, $\tilde{T}=-\tilde{\rho}+3 \tilde{p} \approx-\tilde{\rho}<0$, and appropriate negative values of $\beta_{H}$ provide a negative effective vector mass-square $m_{\text {eff }}^{2}=\left(-4 \pi \beta_{H} \tilde{T} A_{H}^{4}+1\right)$ $e^{2} \bar{\Phi} \Phi$. This means the linearized EOM is tachyonic for $4 \pi \beta_{H} \tilde{T} A_{H}^{4}>1$ thanks to the nonzero value of $\bar{\Phi} \Phi$. The construction respects the gauge symmetry [Eq. (18)] in its explicit form. We call this theory "Higgs-based spontaneous vectorization."

There is one issue that needs elaboration in the above explanation. Remember that the pure Higgs EOM, Eq. (21), is already tachyonic around $\Phi=0$. However, the nonminimal coupling terms bring another factor in front of $m_{0}^{2}$ in Eq. (24), $\left(-4 \pi \beta_{H} \tilde{T} A_{H}^{4}+1\right)^{-1}$, which is negative if we want $X^{\mu}$ to spontaneously grow. Thus, the tachyonic instability of $\Phi$ is eliminated wherever $X^{\mu}$ is tachyonic. This might suggest, at first look, that $\bar{\Phi} \Phi$ does not attain nonzero equilibrium values since the scalar never spontaneously 
grows from $\Phi=0$; hence, the effective tachyonic vector mass term $e^{2} \bar{\Phi} \Phi$ also vanishes, meaning there is no Higgs mechanism for $\Phi$ or spontaneous growth of $X^{\mu}$. This is $n o t$ the case. $\Phi$ is continuous, and if it attains a nonzero value due to an instability in any region of spacetime, it generically attains nonzero values everywhere. For example, in the spontaneous scalarization theory of Eq. (1), the tachyon strictly lives inside the NS, but the scalar field is nonzero everywhere, not just inside the NS. $\phi$ merely dies off away from the star, and it vanishes only at spatial infinity for generic solutions. This means, since the Higgs mechanism is intact in vacuum in Eq. (24), $\bar{\Phi} \Phi$ still attains nonzero values where there is matter even though $\Phi$ is not tachyonic there. Overall, $X^{\mu}$ retains its tachyonic modes and spontaneously grows.

The $X^{\mu}$ terms in the EOM [Eq. (24)] also behave as masssquare terms at the nonperturbative level since $X^{\mu}$ also attains nonzero equilibrium values when it grows spontaneously. These terms might enhance or inhibit the instability of $\Phi$ around its zero value depending on their overall sign, but the same continuity argument can be applied to them as well. At equilibrium, generically $\bar{\Phi} \Phi \neq 0$, which is enough for the spontaneous growth of $X^{\mu}$.

A second form of gauge symmetry-respecting spontaneous growth theory exists which utilizes spontaneous growth itself to provide a nonzero equilibrium value for the scalar. The role of $V(\bar{\Phi} \Phi)$ is shifting the equilibrium value of $\Phi$ away from zero, but tachyon-based spontaneous scalarization already achieves this locally inside NSs, which is where we need it. Consider the action

$$
\begin{aligned}
& \frac{1}{16 \pi} \int d V R-\frac{1}{16 \pi} \int d V F^{\mu \nu} F_{\mu \nu}-\frac{1}{16 \pi} \int d V 2 \overline{D_{\mu} \Phi} D^{\mu} \Phi \\
& \quad+S_{m}\left[\psi_{m}, A_{H}^{2} g_{\mu \nu}\right]
\end{aligned}
$$

where

$$
A_{H}=\exp \left(\frac{\beta_{H}}{2} \overline{D_{\mu} \Phi} D_{\mu} \Phi-\frac{\beta_{H} m_{\Phi}^{2}}{2} \bar{\Phi} \Phi\right)
$$

and $\beta_{H}, m_{\Phi}$ are real constant parameters of the theory. The EOMs are

$$
\begin{aligned}
G_{\mu \nu}= & 8 \pi T_{\mu \nu}+T_{\mu \nu}^{\Phi, X} \\
\nabla^{\nu} F_{\nu \mu}= & \left(-4 \pi \beta_{H} \tilde{T} A_{H}^{4}+1\right)\left(e^{2} \bar{\Phi} \Phi X_{\mu}+J_{\mu}^{\Phi}\right) \\
\square \Phi= & {\left[4 \pi \beta_{H} \tilde{T} A_{H}^{4}\left(1-4 \pi \beta_{H} \tilde{T} A_{H}^{4}\right)^{-1} m_{\Phi}^{2}\right.} \\
& \left.+e^{2} X_{\mu} X^{\mu}+2 i e X^{\mu} \nabla_{\mu}+i e \nabla_{\mu} X^{\mu}\right] \Phi
\end{aligned}
$$

where $T_{\mu \nu}, T_{\mu \nu}^{\Phi, X}$, and $J^{\Phi}$ are as in Eq. (25) but with the new definition of $A_{H}$ in Eq. (27).

We can repeat our previous line of thought and easily see that Eq. (28) shows spontaneous growth of $\Phi$ and $X^{\mu}$. Moreover, this time the mass-square term on the first line of the EOM for $\Phi$ is manifestly tachyonic for $4 \pi \beta_{H} \tilde{T} A_{H}^{4}>1$.
For example, when $4 \pi \beta_{H} m_{\Phi}^{2} \tilde{T} A_{H}^{4} \ll-1$, this term becomes $-m_{\Phi}^{2}$ in the leading order; hence, we can see that the nonzero equilibrium value of $\bar{\Phi} \Phi$ is attained by its own spontaneous growth in the presence of matter, but not as a side effect of tachyonic modes elsewhere in the spacetime.

An interesting aspect of Eq. (27) is that the part that normally causes tachyon-based spontaneous growth, $-\beta_{H} m_{\Phi}^{2} \bar{\Phi} \Phi / 2$ has the wrong sign, it generates the leftmost $4 \pi \beta_{H} \tilde{T} A_{H}^{4}$ term in the EOM of $\Phi$, and does not cause spontaneous growth by itself. However, the $\beta_{H} \overline{D_{\mu} \Phi} D_{\mu} \Phi / 2$ term in $A_{H}$ causes ghost-based spontaneous scalarization through the factor $\left(1-4 \pi \beta_{H} \tilde{T} A_{H}^{4}\right)^{-1}$ at the same time, and only the combination of the two provides the negative mass-square term of $\Phi$ in Eq. (28).

To summarize, $\Phi$ spontaneously grows in Eq. (26) if there is matter, which in turn leads to the spontaneous growth of $X^{\mu}$ through the tachyonic term $m_{\text {eff }}^{2}=$ $\left(-4 \pi \beta_{H} \tilde{T} A_{H}^{4}+1\right) e^{2} \bar{\Phi} \Phi$. The construction again respects the gauge symmetry, which is only spontaneously broken through the nonzero equilibrium value of $\Phi$. One difference from the theory in Eq. (22) is that $X^{\mu}$ becomes asymptotically massless away from the matter in this case, while it is massive in the latter.

Equation (26) is reminiscent of the recently introduced idea of the "gravitational Higgs mechanism" where spontaneous scalarization leads to nonzero equilibrium value for the scalar which in turn generates real mass values for gauge fields [8]. Due to this similarity, we call this phenomenon "gravitational Higgs-based spontaneous vectorization." However, we use spontaneous scalarization terms to incite the growth of the scalar field itself only, and the tachyonic modes of the gauge field $X^{\mu}$ are due to the derivative terms in Eq. (27) in contrast to [8].

We should also emphasize that $\Phi$ in Eq. (22) is not the Higgs field of the Standard Model. $\Phi$ and $X^{\mu}$ can both be better viewed as fundamental degrees of freedom associated with gravity that are hitherto unobserved. However, their possible association to the Standard Model is an interesting research question to study. ${ }^{4}$

Once the fundamental elements of gauge symmetryrespecting spontaneous growth are in place, we can also apply it to more complex field theories. Without going into details, a theory of spontaneously growing non-Abelian gauge fields $W_{\mu}^{a}$, e.g., Yang-Mills theory, is given by the action

$$
\begin{aligned}
& \frac{1}{16 \pi} \int d V R-\frac{1}{16 \pi} \int d V\left(2\left(D_{\mu} \Phi\right)^{\dagger} D^{\mu} \Phi+2 V\left(\Phi^{\dagger} \Phi\right)\right) \\
& -\frac{1}{16 \pi} \int d V F^{a \mu \nu} F_{\mu \nu}^{a}+S_{m}\left[\psi_{m}, A_{\mathrm{YM}}^{2} g_{\mu \nu}\right],
\end{aligned}
$$

\footnotetext{
${ }^{4}$ We are overlooking the fact that the Higgs field of the Standard Model is not a single complex scalar, which is not central to our point.
} 
where all quantities are defined as in Eq. (10) and $\dagger$ indicates a Hermitian conjugate. The Higgs field is now a multidimensional object that can be acted upon by $T^{a}$, and

$$
D_{\mu} \Phi=\nabla_{\mu} \Phi-i e W_{\mu}^{b} T^{b} \Phi .
$$

In analogy to Eq. (22),

$$
V\left(\Phi^{\dagger} \Phi\right)=\frac{1}{2} \frac{m_{0}^{2}}{u^{2}}\left(u^{2}-\Phi^{\dagger} \Phi\right)^{2}
$$

and

$$
A_{\mathrm{YM}}=\exp \left(\frac{\beta_{\mathrm{YM}}}{2}\left(D_{\mu} \Phi\right)^{\dagger} D^{\mu} \Phi\right)
$$

Similarly, we can have the alternative formulation of gravitational Higgs-based spontaneous growth of $W_{\mu}^{a}$ if $V\left(\Phi^{\dagger} \Phi\right)=0$, and

$$
A_{\mathrm{YM}}=\exp \left(\frac{\beta_{\mathrm{YM}}}{2}\left(D_{\mu} \Phi\right)^{\dagger} D^{\mu} \Phi-\frac{\beta_{\mathrm{YM}} m_{\Phi}^{2}}{2} \Phi^{\dagger} \Phi\right)
$$

for appropriate constants $\beta_{H}$ and $m_{\Phi}$.

We will not attempt to numerically find the structures of NSs with spontaneously grown Abelian or non-Abelian gauge fields in this study, but we will discuss certain aspects of such solutions in the final section. EOMs can be easily reduced to a set of coupled ordinary differential equations similar to Tolman-Oppenheimer-Volkoff equations following standard algebra $[9,14]$.

\section{DISCUSSION}

We presented a new form of spontaneous growth mechanism in gravity for gauge fields where the Higgs mechanism is employed to avoid explicit symmetry breaking in the action. Using the Higgs mechanism is a natural choice since the prototypical spontaneous growth theory, spontaneous scalarization, also relies on a mass generation mechanism much like Higgs. We utilize this fact to devise two different theories where the nonzero equilibrium value of the Higgs scalar can arise from a potential term or spontaneous scalarization of the Higgs field.

The Higgs mechanism also leads to modifications in the EOM of the Higgs scalar itself. The effective mass of $\Phi$ necessarily diverges in Eq. (24) and (28) for astrophysical systems due to the $\left(1-4 \pi \beta_{H} \tilde{T} A_{H}^{4}\right)^{-1}$ terms. This is because $\tilde{T}$ vanishes in vacuum, and consequently $1-4 \pi \beta_{H} \tilde{T} A_{H}^{4}=1$ outside a star, whereas $\left(1-4 \pi \beta_{H} \tilde{T} A_{H}^{4}\right)<0$ has to be satisfied inside if there is to be a spontaneous growth of $X^{\mu}$ at all. This means $\left(1-4 \pi \beta_{H} \tilde{T} A_{H}^{4}\right)^{-1}$ necessarily diverges at some points inside a star that experiences Higgs-based spontaneous vectorization.
Such divergent terms are familiar from ghost-based spontaneous growth theories where they have been examined in more detail [9]. Even though there are infinities in the EOM, physical quantities are still finite and continuous, but there are cusps in the density profiles of stars if they spontaneously grow fields this way. It is likely that such features are relatively easy targets for observation, and will lead to strong restrictions in the parameter space of Higgsbased spontaneous growth theories. Strictly tachyon-based theories in Eqs. (3) and (10)-(12) do not have this problem, and likely lead to more regular NS structures. Our current level of knowledge suggests that gauge symmetry and smooth NS structures cannot be achieved in the same theory. Future studies of NSs and their mergers will be the final arbiter of the merit of these two types of theories relative to each other and in comparison to GR.

One difference between the two Higgs mechanisms we investigated is that plain Higgs [Eq. (22)] provides a nonzero $X^{\mu}$ mass everywhere, whereas gravitational Higgs [Eq. (26)] does not generate mass as one goes away from the spontaneously growing field, i.e., the NS. The parameter space for spontaneous scalarization is known to be severely restricted for massless scalars due to measurements from nonmerging binary systems $[10,15]$. This in turn strongly suggests that spontaneous growth of massless Abelian or non-Abelian gauge theories are also at odds with existing tests of gravity. Hence, we can say that the gravitational Higgs-based spontaneous growth is unfavored, but not ruled out, by observations. We leave the quantitative analysis of this issue to future studies.

We have not discussed the regularization of the instabilities we have shown to exist in gauge-field theories. Namely, an instability is not a desired feature in a theory of nature unless it stops growing by additional mechanisms. The fourth-order term of the Higgs potential $V$ that makes $\Phi$ reach equilibrium at finite values, and the suppressive effect of the $A^{\beta \phi^{2} / 2}$ on the tachyonic terms of Eq. (2) as $\phi$ grows are such mechanisms. The arguments in the exponent of $A_{H}$ are not negative definite in either of the two Higgs-based spontaneous growths. Thus, it is not clear which part of the more complicated EOMs Eqs. (24) and (28) can make sure that the instabilities eventually shut off and lead to stable gauge-field clouds. Understanding the stability of the final NS configurations with these growth mechanisms requires detailed numerical studies. It is noteworthy that such questions about the fate of the instability are not easily answered for any generalization of tachyon-based spontaneous scalarization. This statement is true for tachyon-based spontaneous vectorization and ghost-based spontaneous scalarization theories as well, for which numerical solutions have been constructed [5,9].

One may argue that, mathematically, $\overline{D_{\mu} \Phi} D^{\mu} \Phi$ simply hides an $X_{\mu} X^{\mu}$ term, and is not really different from tachyonbased spontaneous vectorization. We can partially embrace such a comment since this is the core of the Higgs 
mechanism: it contains a hidden effective mass term, but in such a way that the gauge symmetry is intact in the action. Moreover, the Higgs mechanism also provides a unified mechanism that generates mass for various fields at the same time as in the Standard Model. This means one can actually devise theories where all fields are spontaneously grown in the presence of matter by a single Higgs kinetic term in the nonminimal matter coupling. However, we restate that we do not identify any of the spontaneously growing fields in this study with known particles of the Standard Model.

Higgs fields are also the source of the mass of spinor fields in the Standard Model, but we have not discussed a Higgs-based spontaneous spinorization theory. This is because spinor masses are generated through the Yukawa mechanism through terms that schematically look like $\bar{\psi} \Phi \psi$ that are linear, not quadratic, in $\Phi[6,7]$. Changing the sign of this term does not provide an instability unlike the gauge fields we considered which have $\bar{\Phi} \Phi$ terms. Spontaneous spinorization theories have been introduced by other means [16], but we believe it would be valuable to find a Higgs-based formulation for spinors as well to demonstrate the universality of the Higgs field to generate tachyonic instabilities as well as mass.

Symmetry is a central concept in modern physics. The main aim of this study was a new formulation of spontaneous growth which would better reflect the fundamental symmetries of nature. We believe the theories we introduced constitute important near-future targets for physics of strong gravity and gravitational wave science.

\section{ACKNOWLEDGMENTS}

We are grateful to Bayram Tekin for stimulating discussions. The author is supported by Grant No. 117F295 of the Scientific and Technological Research Council of Turkey (TÜBİTAK). We would like to acknowledge networking and travel support by the COST Action CA16104.
[1] Y. Fujii and K. Maeda, The Scalar-Tensor Theory of Gravitation (Cambridge University Press, Cambridge, England, 2007).

[2] T. Damour and G. Esposito-Farèse, Phys. Rev. Lett. 70, 2220 (1993).

[3] C. M. Will, Living Rev. Relativity 9, 3 (2006).

[4] E. Berti et al., Classical Quantum Gravity 32, 243001 (2015).

[5] F. M. Ramazanoğlu, Phys. Rev. D 96, 064009 (2017).

[6] P. Frampton, Gauge Field Theories (Wiley, New York, 2008).

[7] M. Srednicki, Quantum Field Theory (Cambridge University Press, Cambridge, England, 2007).

[8] A. Coates, M. W. Horbartsch, and T. P. Sotiriou, Phys. Rev. D 95, 084003 (2017).
[9] F. M. Ramazanoğlu, Phys. Rev. D 97, 024008 (2018).

[10] F. M. Ramazanoğlu and F. Pretorius, Phys. Rev. D 93, 064005 (2016).

[11] N. Arkani-Hamed, H.-C. Cheng, M. A. Luty, and S. Mukohyama, J. High Energy Phys. 05 (2004) 074.

[12] J. B. Jimenez, A. L. D. Froes, and D. F. Mota, Phys. Lett. B 725, 212 (2013).

[13] H. Ruegg and M. Ruiz-Altaba, Int. J. Mod. Phys. A 19, 3265 (2004).

[14] N. Franchini, A. Coates, and T. P. Sotiriou, Phys. Rev. D 97, 064013 (2018).

[15] J. Antoniadis et al., Science 340, 1233232 (2013).

[16] F. M. Ramazanoğlu, Phys. Rev. D 98, 044011 (2018). 